\section{mRCC: Wann Therapieabbruch wegen Toxizität?}

In einer Studie wurden Risikofaktoren für Toxizitäten unter Anti-VEGFTherapien bei Patienten mit mRCC gesucht und außerdem ein Modell zur Vorhersage Toxizitäts-bedingter Therapieabbrüche entwickelt.

nhibitoren des VEGF(„vascular endothelial growth factor“)-Signalwegs sind Standardtherapien beim metastasierten Nierenzellkarzinom (mRCC), aber Toxizitäten können zu Dosisreduktionen, Therapieunterbrechungen oder -abbrüchen führen und damit die Prognose be-

einträchtigen. Untersucht wurden 936 Patienten mit mRCC aus dem International Metastatic Renal Cell Carcinoma Database Consortium, die in der Erstlinie eine Anti-VEGF-Therapie erhalten hatten, $77 \%$ von ihnen Sunitinib. Die mediane Therapiedauer betrug insge-

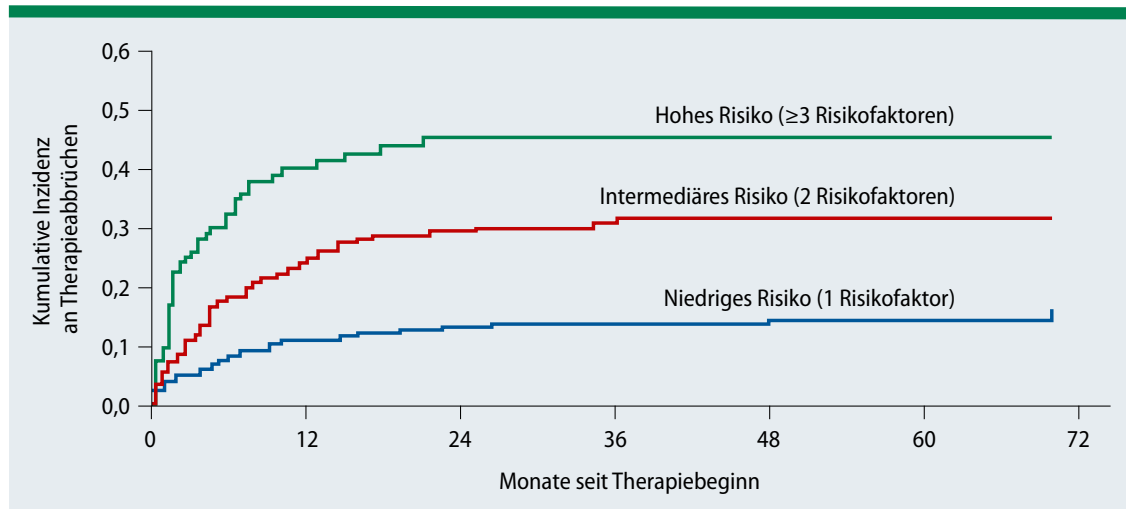

Abb. 1: Das Risiko für einen Therapieabbruch steigt mit der Zahl der Risikofaktoren. samt 7,1 Monate und 4,4 Monate bei Toxizitäts-bedingten Therapieabbrüchen.

833 Patienten hatten die Therapie abgebrochen, $23,8 \%$ wegen Toxizitäten. Die kumulative 1-Jahres-Inzidenz dafür betrug $17 \%$ unter Sunitinib und $23 \%$ unter Sorafenib. Häufigste zu Therapieabbrüchen führende Toxizitäten waren Fatigue (4\%), Diarrhö (2\%) und Appetitmangel (2\%). In einer multivariaten Analyse waren 4 Faktoren prädiktiv für Abbrüche: glomeruläre Filtrationsrate $(\mathrm{GFR})<30 \mathrm{ml} / \mathrm{min} / 1,73 \mathrm{~m}^{2}(\mathrm{p}=0,122)$, solitäre Metastasen $(p=0,096)$, Natriumspiegel $<135 \mathrm{mmol} / \mathrm{l}(\mathrm{p}=0,0056)$ und Alter $\geq 60$ Jahre $(p=0,0022)$. In einem Vorhersagemodell hing das Abbruchrisiko von der Zahl der Risikofaktoren ab (Abb. 1).

Fazit: Bei der Anti-VEGF-Therapie des mRCC sind Alter, GFR, Metastasenzahl und Natriumspiegel unabhängige Risikofaktoren für Abbrüche. Judith Neumaier

Kaymakcalan MD et al. Risk Factors and Model for Predicting Toxicity-Related Treatment Discontinuation in Patients With Metastatic Renal Cell Carcinoma Treated With Vascular Endothelial Growth Factor-Targeted Therapy: Results From the International Metastatic Renal Cell Carcinoma Database Consortium. Cancer. 2016;122(3):411-9.

\title{
Therapieresistentes RCC: Nivolumab verlängert Gesamtüberleben
}

Offene Studien mit dem PD-1-Inhibitor Nivolumab zeigen Erfolge beim mehrfach vorbehandelten Nierenzellkarzinom (RCC). Nun wurde Nivolumab randomisiert gegen die etablierte Drittlinien-Substanz Everolimus getestet.

$\mathrm{D}$ er mTOR-Inhibitor Everolimus ist derzeit Mittel der Wahl für Patienten mit fortgeschrittenem RCC, die gegen Inhibitoren des VEGF(,vascular endothelial growth factor")-Signalwegs wie Sorafenib oder Sunitinib resistent sind. Everolimus verlängert das progressionsfreie Überleben (PFS) der Patienten um einige Monate, was allerdings nicht wirklich einen Durchbruch bedeutet. Möglicherweise hat der $\mathrm{PD}($,programmed cell death protein")-1-Inhibitor Nivolumab größere Chancen. Er verhindert die Interaktion von PD-1 und seinen Liganden, die normalerweise zu einer Hemmung der zellulären Immunantwort führt. In einer Phase-III-Studie wurden Nivolumab und Everolimus verglichen.

Einbezogen waren 821 Patienten mit fortgeschrittenem, klarzelligem RCC, die mindestens 2 antiangiogenetische Therapieregimes durchlaufen hatten. Sie erhielten randomisiert $3 \mathrm{mg} / \mathrm{kg}$ Nivolumab i.v. alle 2 Wochen oder $10 \mathrm{mg} / \mathrm{d}$ Everolimus. Primärer Endpunkt war das Gesamtüberleben (OS). Patienten der Nivolumab-Gruppe lebten deutlich länger als Patienten der Everolimus-Gruppe (25,0 vs. 19,6 Monate; Hazard Ratio 0,73; $\mathrm{p}=0,002)$. Damit war das präspezifizier- te Kriterium für Überlegenheit erfüllt. Auch die Ansprechrate war unter Nivolumab höher ( 25 vs. $5 \%$; p < 0,001). Dabei erwies sich Nivolumab mit schweren Nebenwirkungen bei $19 \%$ der Patienten (hauptsächlich Fatigue) gegenüber Everolimus mit schweren Nebenwirkungen bei $37 \%$ (hauptsächlich Anämie) als besser verträglich.

Fazit: Patienten mit fortgeschrittenem RCC und Resistenzen auf eine VEGFInhibitor-Vortherapie lebten unter $\mathrm{Ni}$ volumab länger als unter Everolimus bei gleichzeitig besserer Verträglichkeit des Checkpointhemmers.

Barbara Kreutzkamp

Motzer RJ et al. Nivolumab versus everolimus in advanced renal-cell carcinoma. N Engl J Med. 2015;373(19):1803-13. 